[Agr. Biol. Chem., Vol. 35, No. 7, p. 1074 1083, 1971]

\title{
Properties and Structures of Peroxidase Isoenzymes of Japanese-radish ${ }^{\dagger}$
}

\author{
By Yuhei MortTa, Chiaki Yoshida and Yutaka MaEdA* \\ The Research Institute for Food Science, Kyoto University, Kyoto \\ * Research Reactor Institute, Kyoto University, Osaka \\ Received January 16, 1971
}

\begin{abstract}
Physicochemical and enzymatic properties of five purified isoenzymes of Japanese-radish peroxidase were investigated comparatively. The molecular weight of peroxidase was different among the acidic, neutral and basic isoenzymes, while the ordered secondary structures seemed to be essentially the same on the observation of infrared spectra. The acidic isoenzyme, No. 3, exhibited a characteristic behavior in the visible absorption spectrum, which showed a thermal interconversion between high- and low-spin states at low temperatures and also the lowest $\mathrm{p} K$ value of the proton dissociation to form the hydroxide compound. The basic isoenzyme, No. 16, was characteristic in its highest affinity for cyanide. In the catalytic activity, the weakly acidic isoenzyme No. 5 was the most active, exhibiting the highest rate constants both for hydrogen peroxide and for guaiacol.
\end{abstract}

In a previous paper, ${ }^{11}$ we demonstrated the peroxidase isoenzymes of Japanese-radish (Raphanus sativus L.) by means of polyacrylamide gel electrophoresis and ion-exchange chromatography on CM- and DEAE-Sephadex. Japanese-radish contains eighteen isoenzymes of peroxidase distinguishable on the electropherograms, among which an acidic isoenzyme (the No. 3 component from the anodic side) is present most abundantly. The earlier preparation of crystalline Japanese-radish peroxidase a $(J R P-a)^{2)}$ was found to contain the major isoenzyme No. 3 predominantly, with a little contamination of other isoenzymes. On the other hand, a basic isoenzyme, named earlier as JRP-c, ${ }^{3,4}$ ' was found to consist of a single component, the No. 16 isoenzyme, on

† Studies on Phyto-peroxidase. Part XXV.

1) Y. Morita, C. Yoshida, I. Kitamura and S. Ida, Agr. Biol. Chem., 34, 1191 (1970).

2) Y. Morita and K. Kameda, Mem. Research Inst. Food Sci., Kyoto Univ., 12, 14 (1957).

3) Y. Morita and K. Kameda, ibid., 12, 1 (1957).

4) Y. Morita, K. Kameda and M. Mizuno, Agr. Biol. Chem., 25, 136 (1961). the electropherogarm.

It is of interest to elucidate the characteristics of the isoenzymes comparatively. In earlier investigations by Morita and his collaborators, ${ }^{5 \sim 71}$ two crystalline preparations, JRP-a and -c, have been characterized comparatively. These enzyme preparations were found to have different chemical compositions and different enzymatic and physicochemical properties from each other. However, it should be noticed that some of these earlier experimental results seem to have possible faults derived from the enzyme preparations used: (1) crystalline JRP-a prepared at lower saturations of ammonium sulfate (less than 0.53 saturation) concentrated the neutral isoenzymes, ${ }^{11}$ and (2) crystalline JRP-c preparation contained a dissociable ligand of low molecular weight, pos-

5) Y. Morita and K. Kameda, Mem. Research Inst. Food Sci., Kyoto Univ., 23, I (1961); 24, 1 (1962).

6) Y. Morita, K. Kameda and M. Mizuno, Agr. Biol. Chem., 26, 442 (1962).

7) K. Shimizu and Y. Morita, ibid., 30, 149 (1966). 
sibly cyanide, unless it was treated by $p$ chloromercuribenzoate (PCMB) or heavy metal ions. ${ }^{8}$ Accordingly it is worthwhile to reinvestigate properties of the pure isoenzymes comparatively.

The present paper demonstrates the comparative characterization of some typical isoenzymes of JRP isolated as single component by ion-exchange chromatography and polyacrylamide gel electrophoresis and a comparison with the isoenzymes of horseradish peroxidase (HRP). ${ }^{9 \sim 11}$

\section{MATERIALS AND METHODS}

JRP isoenzymes. The isolation and purification of JRP isoenzymes were described in the previous report. ${ }^{11}$ Five isoenzyme preparations, i.e. Fractions $\mathrm{A}-1 \mathrm{a}, \mathrm{N}-1$, $\mathrm{N}-2$, JRP-c and the less basic c component, were used in the present experiments. Fractions A-1a, N-1, JRP-c and the less basic component contained a single different isoenzyme in each, No. 3, No. 5, No. 16 and No. 15 component, respectively. The No. 3, No. 15 and No. 16 components were purified in crystalline state. On the other hand, Fraction $\mathrm{N}-2$ was not homogeneous. It contained the neutral isoenzymes, No. 8, 9, 10,11, 12 and 13 components, and the No. 12 component was most abundant. Each of these preparations was treated with PCMB to remove the dissociable ligand ${ }^{8}$ and further purified by gel filtration chromatography on "Sephadex G-75 column, $2.5 \times 90 \mathrm{~cm}$, to remove contaminated impurities as much as possible. The apoenzymes of isoenzyme No. 3 and No. 16 were prepared by acid-acetone treatment at $-10^{\circ} \mathrm{C}$ according to the method of Gjessing and Sumner.12) The apoenzymes were dis-

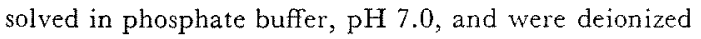
by a Sephadex G-25 column and lyophilized.

8) Y. Morita, C. Yoshida and S. Ida, Agr. Biol. Chem., 33, 436 (1969).

9) L. M. Shannon, E. Kay and J. Y. Lew, J. Biol. Chem., 241, 2166 (1966).

10) E. Kay, L. M. Shannon and J. Y. Lew, ibid., 242, 2470 (1967).

11) E. H. Strickland, E. Kay, L. M. Shannon and J. Horwitz, ibid., 243, 3560 (1968).

12) E. C. Gjessing and J. B. Sumner, Arch. Biochem., 1, 1 (1943).
Peroxidase activity. Two rate constants, $k_{1}$ for hydrogen peroxide and $k_{4}$ for guaiacol, were determined spectrophotometrically according to the method of Chance and Maehly ${ }^{13}$ at room temperature.

Spectrophotometry. Spectrophotometric measurement was perforemd with a Beckman DU spectrophotometer and a Hitachi EPS-2 recording spectrophotometer. Visible absorption spectra at low temperatures were measured with a Shimadzu MPS-50L multipurpose recording spectrophotometer, equipped with a $0.1 \mathrm{~cm}$ light path cell. The cell was placed in a Dewar holding liquid nitrogen. Temperature was varied by introducing nitrogen gas into the Dewar.

Titration experiment. Titration experiment for determining the dissociation constants of cyanide and hydroxide compounds of peroxidase were performed spectrophotometrically at room temperature according to the method described previously. ${ }^{8,14}$

Gel filtration. Gel filtration chromatography for the determination of the molecular weight of the isoenzyme was performed on a Sephadex G-75 column, $2.5 \times 90 \mathrm{~cm}$, according to the method of Andrews. ${ }^{15}$ For the elution of proteins, $0.2 \mathrm{M}$ phosphate buffer, $\mathrm{pH} 7.0$, was used. As marker proteins, following proteins of known molecular weight were purchased and used without further purification. Crystalline bovine serum albumin, hen's egg-white lysozyme, bovine pancreas ribonuclease and horse heart cytochrome $c$ (Type II) from Sigma Chemical Company, St. Louis, Missouri; horse heart metmyoglobin from Calbiochem, Los Angeles, California; and crystalline hen's egg albumin from California Corp. Biochemical Research, Los Angeles, California.

Dodecyl sulfate-polyacrylamide gel electrophoresis. For the determination of molecular weight dodecyl sulfatepolyacrylamide gel electrophoresis was performed according to the method of Shapiro et al., ${ }^{16)}$ modified

13) B. Chance and A.C. Maehly, "Methods in Enzymology," Vol. II, ed. by S. P. Colowick and N. O. Kaplan, Academic Press, New York, 1955, p. 764 .

14) Y. Morita and K. Kameda, Mem. Research Inst. Food Sci., Kyoto Univ., 14, 61 (1958).

15) P. Andrews, Biochem. J., 91, 222 (1956).

16) A. L. Shapiro, E. Vinuela and J. V. Maizel, Biochem. Biophys. Res. Commun., 28, 815 (1967) 
by Weber and Osborn.17) Bovine liver catalase and bovine pancreas $\alpha$-chymotrypsinogen A purchased from Sigma Chemical Company, rabbit muscle aldolase from Boehringer, and horse heart metmyoglobin from Calbiochem were used as the molecular weight standards.

Infrared spectroscopy. Infrared spectroscopy of proteins was performed by means of a Hitachi EPI-S2 infrared spectrophotometer, equipped with $0.05 \mathrm{~mm}$ KRS-5 cells. The lyophilized peroxidase isoenzymes and the apoenzymes were dissolved in heavy water (99.75\% $\mathrm{D}_{2} \mathrm{O}$ purchased from E. Merck AG, Darmstadt, Germany) to make about $9 \%$ protein solutions.

\section{RESULTS}

\section{Molecular weights of isoenzymes}

Molecular weights of the JRP isoenzymes were determined by gel filtration chromatography on a Sephadex G-75 column, $2.5 \times 90$ $\mathrm{cm}$, equilibrated with $0.2 \mathrm{M}$ phosphate buffer, $\mathrm{pH}$ 7.0. The semilogarithmic relationship between molecular weight and elution volume is shown in Fig. 1. The major acidic isoenzyme, the No. 3 component, has a molecular weight of 45,000 , which was determined by protohematin content of the purified No. 3 isoenzyme. ${ }^{18)}$ The value fairly fits to the present experimental result in the gel filtration chromatography. The weakly acidic isoenzyme, No. 5, exhibited the same behavior as that of the No. 3 isoenzyme in the gel filtration chromatography. Accordingly both isoenzymes must have the same molecular weight. On the other hand, JRP-c, a basic isoenzyme

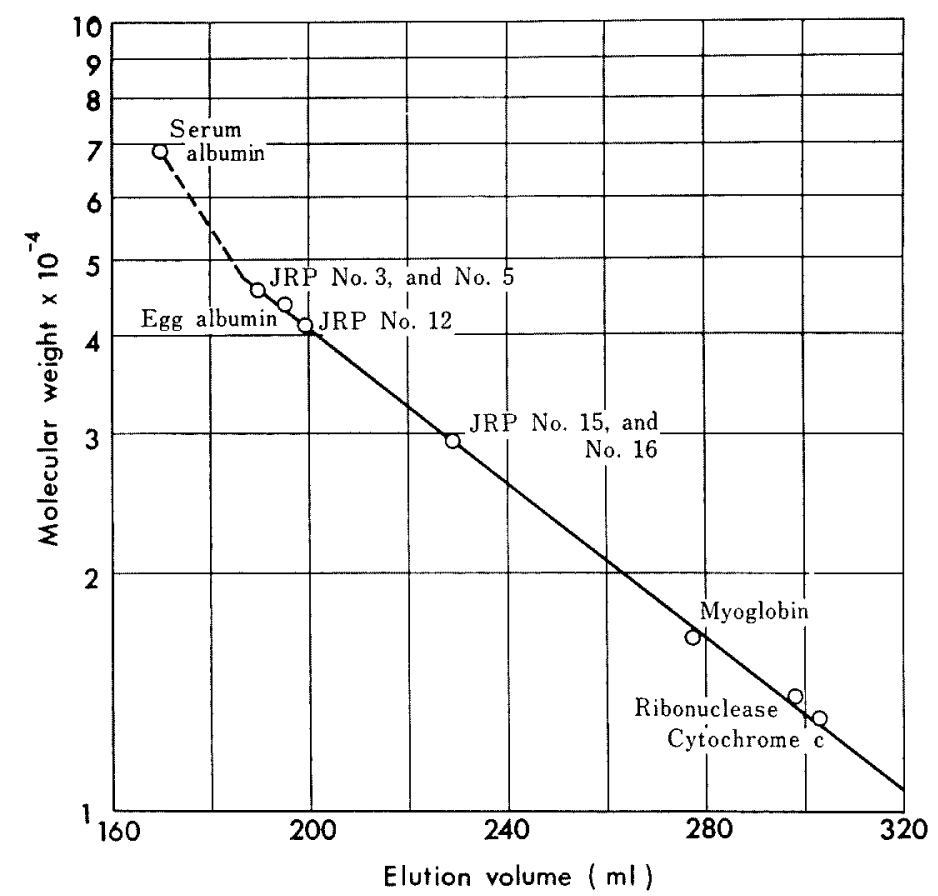

FIG. 1. Determination of Molecular Weights of Peroxidase Isoenzymes by Gel Filtration Chromatography on a Sephadex G-75 Column, $2.5 \times 90 \mathrm{~cm}$.

Experimental details are given in the text.

17) K. Weber and M. Osborn, J. Biol. Chem., 244, 4406 (1969).
18) Y. Morita and C. Yoshida, Agr. Biol. Chem., 34, $590(1970)$. 


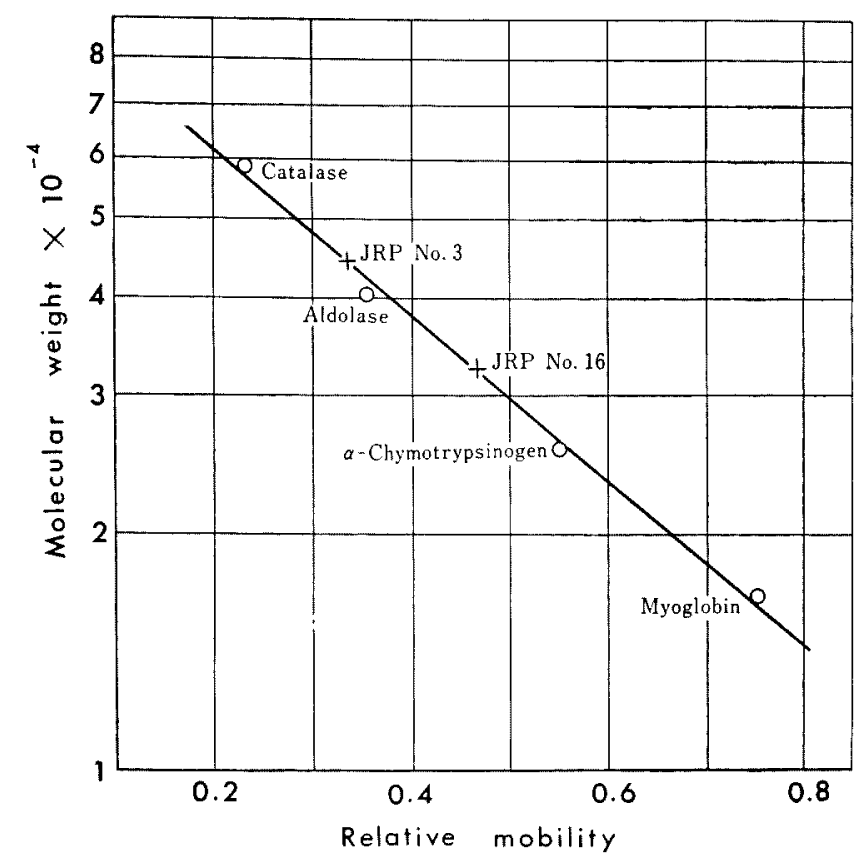

FIG. 2. Determination of Molecular Weights of Peroxidase Isoenzymes by Dodecyl Sulfate-Polyacrylamide Gel Electrophosesis.

Experimental details are given in the text.

denoted as component No. 16, emerged in the gel filtration at the position corresponding to the molecular weight of 30,000. Another method of molecular weight determination, dodecyl sulfate-polyacrylamide gel electrophoresis, also gave the value of 32,000 for the No. 16 component and 44,000 for the No. 3 component, respectively, as shown in Fig. 2. The overestimated value in the earlier experiment for the No. 16 component seems to have been derived from the fault in estimation of the hematin content of the preparation used, which might have been heavily contaminated with inorganic salts due to incomplete dialysis. ${ }^{4 !}$ Since the No. 15 isoenzyme exhibited the same behavior as that of the No. 16 isoenzyme in the gel filtration chromatography, both must have the same molecular weight, while the No. 12 isoenzyme showed a molecular weight of 40,500 , which is similar to that of HRP
II. $^{191}$

Ultraviolet and visible absorption spectra

The light absorption spectra' of the No. 3 and No. 16 components were presented in the previous papers. ${ }^{8,181}$ All spectra of the isoenzymes treated with PCMB are almost indistinguishable in their general patterns, which exhibit the typical high-spin nature; and the pyridine hemochromogen bands reveal that all isoenzymes contain protohematin as the prosthetic group. The molar extinction coefficients at the absorption maxima, determined on the basis of the protohematin concentrations, are listed in Table I, which also contains the values of HRP obtained by Keilin and Hartree ${ }^{19 !}$

19) D. Keilin and E. F. Hartree, Biochem. J., 49, 88 (1951). 
TABLE I. ABSORPtion MAXIMA AND MOLAR EXTINCTION COEFFICIENTS OF Peroxidase Isoenzymes

\begin{tabular}{ccc}
\hline $\begin{array}{c}\text { Peroxidase } \\
\text { isoenzyme }\end{array}$ & $\begin{array}{c}\text { Absorption } \\
\text { maxima } \\
\text { m } \mu\end{array}$ & $\begin{array}{c}\text { Molar extinction } \\
\text { coefficient } \\
\mathrm{mM}^{-1} \mathrm{~cm}^{-1}\end{array}$ \\
\hline JRP No. 3 & 280 & 27.2 \\
& 404 & 111.4 \\
& 502 & 11.70 \\
JRP No. 5 & 644 & 3.23 \\
& 280 & 38.2 \\
& 405 & 116.6 \\
JRP No. I6 & 502 & 11.56 \\
& 644 & 3.38 \\
& 280 & 29.7 \\
& 403 & 104.2 \\
HRP II & & 11.86 \\
& 504 & 3.39 \\
& 645 & 107.7 \\
& 403 & 11.87 \\
& 497 & 3.36 \\
\hline
\end{tabular}

a) From the data of Keilin and Hartree, ${ }^{19}$ ) corrected according to Paul et al.201

and corrected according to Paul et al. ${ }^{20\}}$ The differences in the corresponding values at each absorption maximum are found to be very small between the isoenzymes.

Morita and Mason ${ }^{211}$ stated in an electron paramagnetic resonance study of peroxidase that JRP-a (the acidic isoenzyme) should be in thermal equilibrium between high- and low-spin states. As it was proposed by George et al. ${ }^{22}$ that the visible bands of hemoproteins were characteristic of the spin-states of the heme-iron atom, the authors measured the visible absorption bands of JRP isoenzymes at low temperatures. Figure 3 shows the spectra of the frozen solution of JRP iso-

20) K. G. Paul, H. Theorell and A. Akeson, Acta Chem. Scand., 7, 1284 (1953).

21) Y. Morita and H.S. Mason, J. Biol. Chem., 240, 2654 (1965).

22) P. George, J. Beetlestone and J.S. Griffith, "Haematin Enzymes," ed. by J. E. Falk, R. Lemberg and R.K. Morton, Pergamon Press, Oxford, 1961, p. 105 . enzymes No. 3, No. 5 and No. 16 in $0.03 \mathrm{M}$ phosphate buffer, $\mathrm{pH} 7.0$, at 77 to $250^{\circ} \mathrm{K}$. The No. 5 and No. 16 isoenzyme exhibited almost no essential changes in their visible spectra, while the No. 3 isoenzyme showed a marked change of the band maximum from 495 to $540 \mathrm{~m} \mu$ by lowering the temperature. This alteration of the band maximum should coincide with the alteration in the spin-states of the heme-iron from high-spin to low-spin. It is interesting to note that only the acidic isoenzyme could exhibit such a change. Similar changes in the visible bands at low temperatures were observed also in yeast cytochrome c peroxidase. ${ }^{231}$

\section{Dissociation constants of hydroxide and cyanide compounds}

All of the isoenzymes are able to combine with various extrinsic ligands such as cyanide, hydroxide, azide and fluoride. Among the various compounds of peroxidase, the hydroxide and cyanide are of most interest in distinction of the isoenzymes. ${ }^{8,91}$ Figure 4 shows the dissociation curves of four peroxidase isoenzymes into their hydroxide forms. The $\mathrm{p} K$ values of the proton dissociation of the No. 3, No. 5, No. 12 and No. 16 isoenzymes at ionic strength 0.1 and at $18^{\circ} \mathrm{C}$ are found to be $9.8,10.5$, 10.9 and 10.3, respectively. It is found that the basic isoenzyme, No. 16 , has a higher $\mathrm{p} K$ value than that of acidic No. 3, and the $\mathrm{p} K$ value of the weakly acidic isoenzymes, No. 5 , resembles rather that of the basic one. The neutral isoenzyme, No. 12, has the highest $\mathrm{p} K$ value.

The dissociation constants of the cyanide

23) T. Yonetani, D. F. Wilson and B. Seamonds, J. Biol. Chem., 241, 5347 (1966).

24) D. Keilin and E. F. Hartree, Biochem. J., 61, 153 (1955).

25) I. Yamazaki, R. Nakajima, H. Honma and M. Tamura, "Structure and Function of Cytochromes," ed. by K. Okunuki, M. D. Kamen and I. Sekuzu, Univ. Tokyo Press, 1968, p. 552.

26) T. Yonetani and G. S. Ray, J. Biol. Chem., 240, 4503 (1965). 

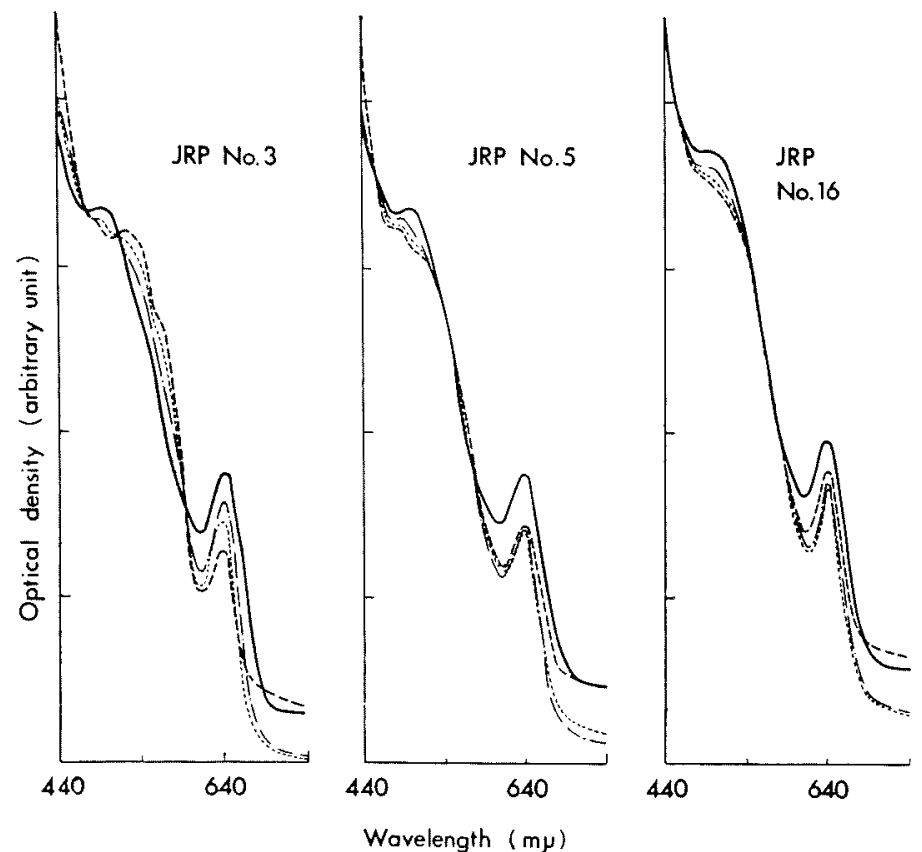

FIG. 3. Visible Absorption Spectra of Peroxidase Isoenzymes at Low Temperatures.

The enzymes were dissolved in $0.03 \mathrm{M}$ phosphate buffer, $\mathrm{pH} 7.0$. Light path: $0.1 \mathrm{~cm}$.

$-250^{\circ} \mathrm{K},-.170^{\circ} \mathrm{K}, \quad 120^{\circ} \mathrm{K},--77^{\circ} \mathrm{K}$.

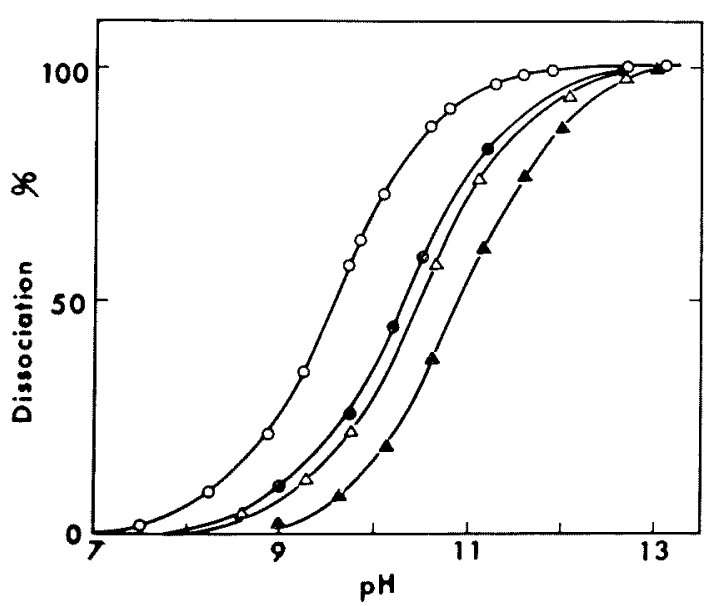

FIG. 4. Dissociation Curves of Peroxidase Isoenzymes to their Alkaline Forms.

O-O No. 3 isoenzyme, - No. 16 isoenzyme, $\triangle-\triangle$ No. 5 isoenzyme, $\Delta$ No. 12 isoenzyme. The titrations were carried out in $0.1 \mathrm{M}$ potassium chloride solutions at $18^{\circ} \mathrm{C}$.
Table II. Dissociation Constants of PeroxidaseCyANide Compounds

The values of JRP isoenzymes were obtained at $20^{\circ} \mathrm{C}$.

\begin{tabular}{|c|c|c|c|c|}
\hline \multirow[b]{2}{*}{ Peroxidase } & \multirow[b]{2}{*}{$\mathrm{pH}$} & \multicolumn{2}{|c|}{ Dissociation constant } & \multirow{2}{*}{ Reference } \\
\hline & & $\begin{array}{l}\text { Oxidized } \\
\text { form }\end{array}$ & $\begin{array}{l}\text { Reduced } \\
\text { form }\end{array}$ & \\
\hline JRP No. 3 & 8.0 & $3 \times 10^{-6}$ & $2 \times 10^{-3}$ & 8 \\
\hline JRP No. 5 & 8.0 & $2 \times 10^{-6}$ & $5 \times 10^{-4}$ & \\
\hline JRP No. 12 & 8.0 & $2 \times 10^{-b}$ & $7 \times 10^{-4}$ & \\
\hline JRP No. 16 & 8.0 & $5 \times 10^{-7}$ & $6 \times 10^{-5}$ & 8 \\
\hline HRP II & 9.15 & $2 \times 10^{-6}$ & $1.3 \times 10^{-3}$ & 24 \\
\hline HRP I & 7.0 & $3 \times 10^{-8}$ & $2 \times 10^{-7}$ & 25 \\
\hline WGPa) 556 & 7.0 & $6 \times 10^{-5}$ & 1 & 25 \\
\hline WGP 566 & 7.0 & $8 \times 10^{-8}$ & $4 \times 10^{-7}$ & 25 \\
\hline $\mathrm{YCP}^{b)}$ & 6.0 & $3 \times 10^{-6}$ & & 26 \\
\hline
\end{tabular}

a) Wheat germ peroxidase.

b) Yeast cytochrome $c$ peroxidase. 
compounds of the isoenzymes are listed in Table II together with those of the previously reported peroxidases. ${ }^{21,231}$ The constants of the acidic and basic isoenzymes were already discussed in the previous paper. ${ }^{81}$ The weakly acidic isoenzyme, No. 5 , and the neutral isoenzyme, No. 12 , have similar values to that of the acidic isoenzyme, No. 3 , in the oxidized forms; but the reduced forms of the former isoenzymes exhibit intermediate values between those of the reduced forms of the acidic No. 3 and the basic No. 16 components. This fact suggests that both the No. 5 and No. 12 isoenzymes will be in the high-spin form free from a ligand even without the pretreatment of PCMB. The basic No. 15 isoenzyme has the entirely same behaviors in cyanide and hydroxide compounds as those of the No. 16 isoenzyme.

\section{Enzymatic activity}

Two rate constants, $k_{1}$ for hydrogen peroxide and $k_{4}$ for guaiacol, of the PCMB-treated JRP isoenzymes were determined from the overall reaction according to the method of Chance and Maehly. ${ }^{13)}$ The values are shown in Table III. It is interesting to note that the values of $k_{1}$ do not vary so much among the isoenzymes, while the $k_{4}$ values are markedly different. Especially the No. 5 component is highly active, exhibiting the $k_{4}$ value 15 times higher than that of the No. 3 isoenzyme. The No. 5 isoenzyme is the most active peroxidase among the JRP isoenzymes tested.

TABLE III. RATE CONSTANTS, $k_{1}$ FOR HydRogen PEROXIDE AND $k_{4}$ FOR GUAIACOL, OF PEROXIDASE ISOENZYMES

All measurements were made at $20^{\circ} \mathrm{C}$.

\begin{tabular}{ccc}
\hline $\begin{array}{c}\text { Peroxidase } \\
\text { isoenzyme }\end{array}$ & $\begin{array}{c}k_{1} \\
\mathrm{M}^{-1} \mathrm{sec}^{-1}\end{array}$ & $\begin{array}{c}k_{4} \\
\mathrm{M}^{-1} \mathrm{sec}^{-1}\end{array}$ \\
\hline JRP No. 3 & $4.2 \times 10^{6}$ & $7.6 \times 10^{4}$ \\
JRP No. 5 & $7.5 \times 10^{6}$ & $1.2 \times 10^{6}$ \\
JRP No. 12 & $2.5 \times 10^{6}$ & $2.5 \times 10^{5}$ \\
JRP No. 16 & $3.6 \times 10^{6}$ & $3.4 \times 10^{5}$ \\
\hline
\end{tabular}

Infrared spectra of peroxidase and apoenzyme

The authors previously reported a circular dichroic study of the acidic isoenzyme showing the ordered structures, i.e., the $\alpha$ helical structure in the holoenzyme and the $\beta$ or pleated sheet structure in the apoenzyme. ${ }^{27)}$ However, the circular dichroism could not distinguish whether the $\beta$ structure in the apoenzyme was originally involved in the holoenzyme or it was formed when the heme was removed from the holoenzyme. Now a more useful technique to show the $\beta$ structure in the protein, infrared spectroscopy, is employed. Figure 5 shows the infrared spectra of two peroxidase isoenzymes, No. 3 and No. 16, and their apoenzymes in heavy water solution at around the wave number of $1650 \mathrm{~cm}^{-1}$, all the spectra show the absorption maxima at about $1630 \mathrm{~cm}^{-1}$, characteristic of the amide I bands in the $\beta$ structure of the protein. ${ }^{28,29)}$ This fact suggests that the $\beta$ structures are originally present in the holoenzymes and remain in the apoenzymes even after the removal of the heme. In the cases of the holoenzymes shoulders can be seen at about $1650 \mathrm{~cm}^{-1}$, while they diminish in the apoenzymes. These bands should be attributed to the $\alpha$ helical structures in the holoenzymes. ${ }^{271}$ Furthermore, it is interesting to indicate the decrease in the absorption of the amide II bands at around $1530 \mathrm{~cm}^{-1}$ in the apoenzymes as compared with the holoenzymes. The decrease must be due to the easier exchange of amide hydrogen with deuterium in the apoenzymes than in the holoenzymes, as in the cases of chymotrypsinogen and lysozyme. ${ }^{301}$ It is of interest to note that the secondary structures of the two different isoenzymes derived from the infrared spectra seem to be essentially the

27) K. Hamaguchi, K. Ikeda, C. Yoshida and Y. Morita, J. Biochem. (Tokyo), 66, 191 (1969).

28) E. J. Ambrose and A. Elliott, Proc. Roy. Soc. London, A205, 47 (1951).

29) A. Elliott, ibid., A221, 104 (1954).

30) E. R. Blout, C. de Lozé and A. Asadourian, J. Am. Chem. Soc., 83. 1895 (1961). 


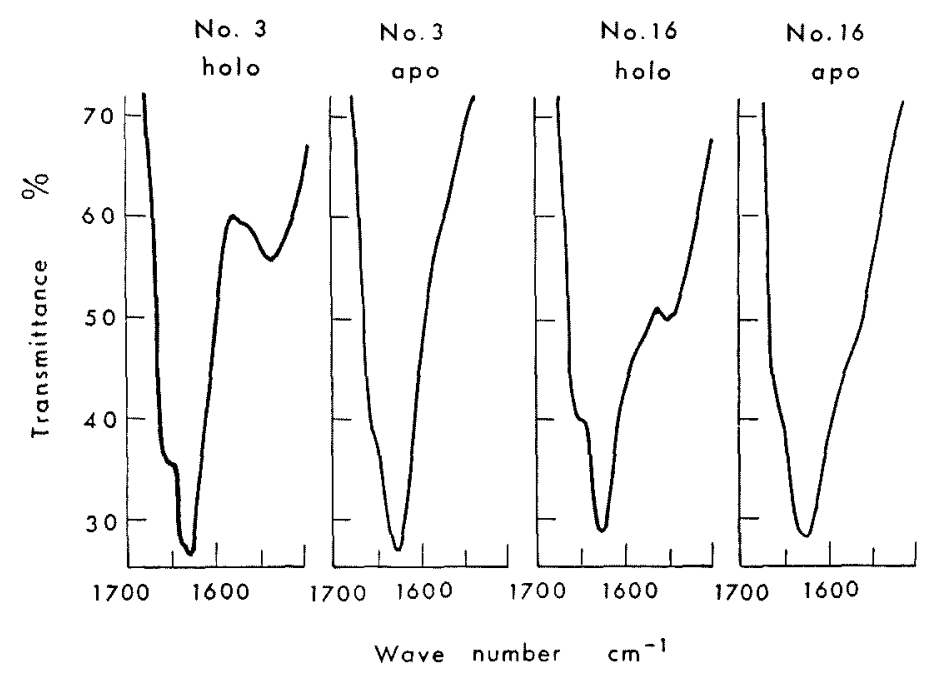

FIG. 5. Infrared Spectra of Peroxidase Isoenzymes, No. 3 and No. 16, and their Apoenzymes in Heavy Water Solutions.

Protein concentration: $10 \mathrm{mg}$ protein in $100 \mu \mathrm{D}_{2} \mathrm{O}$.

Light path: $0.05 \mathrm{~mm}$.

same and they similarly change by the removal of heme.

\section{DISCUSSION}

The differences in some physicochemical properties between JRP isoenzymes, such as the solubility in ammonium sulfate solution and the behaviors on electrophoresis and ionexchange chromatography, were already described in the previous paper. ${ }^{\text {" }}$ The present investigation reveals the differences in other physicochemical and enzymatic properties of JRP isoenzymes, e.g. the affinity of cyanide, the proton dissociation to form the hydroxide compounds, and the rate constants for the hydrogen donor and acceptor. These differences may be derived from the variance in the gross structures of the isoenzymes and their fine structures around the heme-iron atom. Table IV shows the amino acid compositions of two typical JRP isoenzymes, No. 3 and No. 16, together with those of HRP isoenzymes and yeast cytochrome $c$ peroxidase
(YCP) ${ }^{9,311}$ The numbers of amino acid residues per each molecule were obtained by the correction of the previously reported data with respect to the molecular weights and the residue recoveries in the analyses. ${ }^{71}$ The two isoenzymes have markedly different molecular weights, but the difference corresponding to about 7,000 Daltons is due to the carbohydrate content. It is interesting to note that some amino acids are essentially the same or very similar in numbers in both isoenzymes. This fact suggests a genetic relationship between the structural genes producing two different isoenzymes. Some identical and similar values also can be seen between JRP and HRP isoenzymes. A most marked difference in the amino acid compositions between isoenzymes is found in the contents of arginine and hydroxy amino acids. In the circular dichroic study of JRP, ${ }^{271}$ the authors have suggested a relationship between the high content of hydroxy amino acids and the presence of the $\beta$

31) N. Ellfolk, Acta Chem. Scand., 21, 2736 (1967). 
Table IV. Amino Acid Compositions of Peroxidase Isoenzymes

The values are the nearest integers of the amino acid residues per each molecule.

\begin{tabular}{|c|c|c|c|c|c|c|}
\hline \multirow{2}{*}{$\begin{array}{l}\text { Peroxidase } \\
\text { isoenzyme } \\
\text { Molecular weight }\end{array}$} & \multicolumn{2}{|c|}{ JRP } & \multicolumn{3}{|c|}{ HRP } & \multirow{2}{*}{$\begin{array}{l}\text { YCP } \\
34,100\end{array}$} \\
\hline & $\begin{array}{l}\text { No. } 3 \\
45,000\end{array}$ & $\begin{array}{l}\text { No. } 16 \\
32,000\end{array}$ & $\begin{array}{c}\mathrm{A}-1 \\
45,000\end{array}$ & $\underset{40,000}{B}$ & $\underset{40,000}{\mathbf{C}}$ & \\
\hline Aspartic acid & 47 & 36 & 47 & 51 & 52 & 42 \\
\hline Glutamic acid & 24 & 24 & 22 & 20 & 22 & 27 \\
\hline Glycine & 27 & 34 & 24 & 17 & 16 & 23 \\
\hline Alanine & 33 & 28 & 57 & 23 & 23 & 16 \\
\hline Valine & 19 & 27 & 22 & 17 & 17 & 12 \\
\hline Leucine & 33 & 27 & 32 & 36 & 37 & 23 \\
\hline Isoleucine & 14 & 16 & 14 & 12 & 13 & 8 \\
\hline Proline & 16 & 16 & 13 & 16 & 17 & 15 \\
\hline Serine & 47 & 23 & 54 & 23 & 22 & 14 \\
\hline Threonine & 25 & 16 & 47 & 25 & 24 & 14 \\
\hline Hydroxyproline & 11 & 0 & + & 0 & 0 & 0 \\
\hline Half cystine & 8 & 10 & 4 & 4 & 4 & 2 \\
\hline Methionine & 3 & 3 & 1 & 3 & 3 & 8 \\
\hline Phenylalanine & 17 & 14 & 19 & 22 & 23 & 16 \\
\hline Tyrosine & 2 & 2 & 2 & 4 & 5 & 12 \\
\hline Tryptophan & 2 & 2 & 1 & 1 & 1 & 6 \\
\hline Histidine & 4 & 4 & 3 & 3 & 3 & 5 \\
\hline Lysine & 6 & 8 & 6 & 5 & 6 & 21 \\
\hline Arginine & 10 & 30 & 10 & 20 & 21 & 9 \\
\hline Amide ammonia & 46 & 27 & & & & \\
\hline
\end{tabular}

or pleated sheet structure in the acidic isoenzyme molecule, based upon the similarity to the case of poly-L-serine. ${ }^{321}$ But this seems not to be the case, because the present investigation on the infrared spectra reveals the $\beta$ structure in the two isoenzymes containing markedly different amounts of hydroxy amino acids. Strickland et al..$^{9}$ also reported a similarity in the secondary structures of the acidic and neutral isoenzymes of HRP in spite of their different hydroxy amino acid contents.

It is of much interest to note the comparative behavior of the weakly acidic No. 5 isoenzyme. Although its molecular weight, absorption spectrum and the cyanide affinity in the oxidized form resemble those of the acidic No. 3 isoenzyme, the dissociation of proton

32) F. Quadrifoglio and D. W. Urry, J. Am. Chem., Soc., 90, 2760 (1968). to form the hydroxide compound and the cyanide affinity in the reduced form are very different between the two. The enzymatic activity of the No. 5 isoenzyme is much higher than the others, and this high activity resembles that of turnip peroxidase B reported by Hosoya. ${ }^{33)}$ The No. 5 isoenzyme seems to have a higher content of tyrosine, which may be correlated to its higher enzymatic activity. The details will be presented elsewhere. The higher content of tyrosine in the neutral HRP isoenzymes was also reported. ${ }^{11}$

The dissociation of proton from the water molecule at the sixth coordination position of heme-iron atom is remarkably different between the acidic No. 3 isoenzyme and the others. This difference was found also on

33) T. Hosoya, J. Biochem. (Tokyo), 47, 369, 794 (1960). 
HRP isoenzymes by Shannon et al., ${ }^{91}$ and it seems to be correlated with the difference in the spin states of the heme-iron in peroxidase at low temperatures. Morita and Mason $^{21}$ demonstrated by electron paramagnetic resonance spectroscopy that JRP-a in a frozen neutral solution existed in a mixture of highand low-spin states, and at lower $\mathrm{pH}$ the highspin form was abundant. Furthermore, HRP was in more high-spin nature even at the neutral $\mathrm{pH}$ than was JRP. However, we conclude that the magnetic properties are not essentially different between HRP and JRP but the difference rather exists between isoenzymes. JRP-a consisted mainly of the acidic isoenzyme, No. 3, while HRP II consisted mainly of the neutral isoenzymes. Then the difference will be observed between the acidic and the neutral isoenzymes even in HRP or JRP in itself, as shown in Fig. 3. The conflicting results on the Mössbauer spectra between HRP and JRP at low temperatures ${ }^{34,351}$ could have also been derived from using the different isoenzymes.

Acknowledgements. The authors would like to express their thanks to Dr. D. Ida and Mr. I. Kitamura for their kind assistance in the preparation of the enzymes and also for their valuable discussions.

34) Y. Maeda and Y. Morita, "Structure and Function of Cytochromes," ed. by K. Okunuki, M. D. Kamen and 1. Sekuzu, Univ. Tokyo Press, 1968, p. 523.

35) A. J. Bearden, A. Ehrenberg and T. H. Moss, ibid., p. 528 . 